\title{
The effect of selenium and linseed oil on growth of sheep and content of selected fatty acids in M. longissimus dorsi*
}

\author{
M. Czauderna ${ }^{1}$, J. Kowalczyk, K.M. Niedźwiedzka, I. Wąsowska \\ and J.J. Pająk
}

The Kielanowski Institute of Animal Physiology and Nutrition, Polish Academy of Sciences 05-110 Jabtonna, Poland

\begin{abstract}
The influence of linseed oil (LO) and selenium (as $\mathrm{Na}_{2} \mathrm{SeO}_{4}$ ) on organs, muscles and body weight gain of sheep, as well as the fatty acid composition of M. longissimus dorsi was investigated. Twenty male lambs of body weight of $25 \pm 2 \mathrm{~kg}$ were divided into four treatment groups and fed a control diet or experimental diets enriched in 5\% linseed oil (LO), 2 ppm selenium (SE) or combined addition of 5\% LO and $2 \mathrm{ppm}$ selenium (LOSE). The effects of the experimental diets on organs and body weight gain of the sheep were small, but the LOSE treatment increased M. biceps femoris mass and body weight gain. All experimental diets numerically decreased the level of saturated fatty acids as well as the content of cis-9C18:1 in M. longissimus dorsi. LO supplied to the diet numerically increased the content of conjugated linoleic acid isomers and other unsaturated fatty acids in $M$. longissimus dorsi (with the exception of cis-9C18:1). The SE and LOSE treatments numerically decreased the content of unsaturated fatty acids.
\end{abstract}

KEY WORDS: linseed oil, selenium, fatty acids, sheep

\section{INTRODUCTION}

Milk and meat products of ruminant origin are important sources of nutrients in consumer diets, providing some beneficial mono- and polyunsaturated fatty acids (MUFA and PUFA). In numerous experiments it have been found that feeding vegetable oils, fish oils or fish meal rich in PUFA decreased the content of saturated fatty acids (SFA) in meat and milk as well as increased the content of valuable MUFA (cis- and trans-MUFA), PUFA and fatty acids (FA) containing

\footnotetext{
* Supported in part by the State Committee for Scientific Research, Grant No. 3 PO6Z 03422

${ }^{1}$ Corresponding author: e-mail: m.czauderna@ifzz.pan.pl
} 
conjugated double bonds. Consequently the percentage of other undesirable FA decreased. In several studies it were found that the unsaturated fatty acid (USFA) levels were positively correlated with the level of selenium (Se) in the diet. Se is an integral component of antioxidant enzymes (e.g., glutathione peroxidases) that can decrease the risk of USFA peroxidation (Arteel and Sies, 2001). The potential importance of MUFA and PUFA, as well as that of Se, made it important to study the extent to which dietary linseed oil (LO) and Se (as sodium selenate) may increase the USFA content of $M$. longissimus dorsi. The objective of the current study was also to examine the effect of the experimental diets on the body weight gain (BWG) of sheep and mass of muscles and selected organs.

\section{MATERIAL AND METHODS}

Twenty male Polish Merino lambs with an average body weight (BW) of $25 \pm 2$ $\mathrm{kg}$ at the beginning of the experiment were individually penned and divided into 4 treatment groups of 5 animals. During a 1-week preliminary period the animals were given ad libitum access to the standard concentrate-hay diet. Then, for 35 days the sheep were fed the standard concentrate-hay diet (Control) or experimental diets enriched in 5\% linseed oil (LO), 2 ppm Se (SE) or the experimental diet with the combined addition of 5\% LO and $2 \mathrm{ppm}$ Se (LOSE). The rations were adjusted weekly to ensure ad libitum access to feed.

All of the chemicals used, the method of hydrolysis (Czauderna and Kowalczyk, 2001), derivatization, HPLC configurations were described previously (Czauderna et al., 2002, 2004). Underivatized conjugated linoleic acids (CLA) isomers and other FA containing conjugated double bonds (CFA) were determined according to Czauderna et al. (2003), while all derivatized fatty acids were determined according to Czauderna and Kowalczyk (2002). Statistical analyses of the effect of LO or SE treatments were conducted using the nonparametric MannWhitney U test, while statistical analyses of the simultaneous selenium and LO treatment were performed applying two-factorial analysis. The Statistica (version 6) and Excel 2000 programs were used.

\section{RESULTS}

No lesions or symptoms of harmful effects of LO and Se were found in sheep fed the experimental diets. Experimental data on BW, organ weight and composition of selected fatty acids in M. longissimus dorsi after 35 days of feeding the experimental diets are summarized in Table 1. Feeding LO or Se to sheep numerically increased the BW of sheep, while the LOSE treatment elevated BW. Feeding the diet enriched in LO or in LO and Se (LOSE) resulted in numerically increased mass of the liver, lungs, spleen, kidneys, $M$. 
longissimus dorsi and M. biceps femoris. The LO or Se treatment numerically decreased the content of cis-9C18:1, C16:0, C18:0 and the sum of SFA in $M$. longissimus dorsi. The SFA content of $M$. longissimus dorsi decreased $(\mathrm{P}<0.05)$ with LOSE. The LO treatment numerically increased CLA, cis-MUFA and PUFA contents in M. longissimus dorsi (with the exception of cis-9C18:1),

Table 1. Effect of diets on the BW of sheep and organs ${ }^{1}$ and FA content in M. longissimus dorsi ${ }^{2}$

\begin{tabular}{|c|c|c|c|c|c|c|c|c|}
\hline \multirow{2}{*}{ Item } & \multicolumn{4}{|c|}{ Group } & \multirow{2}{*}{$\mathrm{SD}^{3}$} & \multicolumn{3}{|c|}{ Significance of effect ${ }^{3}$} \\
\hline & control & LO & SE & LOSE & & LO & $\mathrm{SE}$ & LOSE \\
\hline Initial body mass ${ }^{4}, \mathrm{~kg}$ & 24 & 24.4 & 24.8 & 25.8 & 2.4 & NS & NS & NS \\
\hline Final body mass ${ }^{5}, \mathrm{~kg}$ & 30.6 & 33.9 & 31.7 & 35.0 & 2.7 & NS & NS & $*$ \\
\hline Liver, $\mathrm{g}$ & 618 & 625 & 563 & 659 & 54 & NS & NS & NS \\
\hline Lungs, $g$ & 643 & 665 & 629 & 652 & 85 & NS & NS & NS \\
\hline Spleen, $g$ & 77 & 88 & 78 & 89 & 16 & NS & NS & NS \\
\hline Pancreas, g & 38 & 33 & 39 & 37 & 7 & NS & NS & NS \\
\hline Kidneys, $g$ & 89 & 97 & 97 & 100 & 9 & $*$ & NS & NS \\
\hline Brain, $g$ & 82 & 87 & 89 & 82 & 5 & NS & NS & NS \\
\hline M. biceps femoris ${ }^{1}, \mathrm{~kg}$ & 4.09 & 4.69 & 4.26 & 4.61 & 0.39 & NS & NS & $*$ \\
\hline M. longissimus dorsi ${ }^{1}, \mathrm{~g}$ & 371 & 417 & 373 & 385 & 57 & NS & NS & NS \\
\hline CLA, $\mu \mathrm{g} / \mathrm{g}$ & 345 & 400 & 204 & 242 & 128 & NS & $*$ & NS \\
\hline $\mathrm{CFA}, \mu \mathrm{g} / \mathrm{g}$ & 108 & 67 & 67 & 80 & 43 & $*$ & NS & NS \\
\hline Cis-9C18:1, mg/g & 3.05 & 2.11 & 2.29 & 1.88 & 0.86 & NS & NS & $*$ \\
\hline $\mathrm{C} 16: 0, \mathrm{mg} / \mathrm{g}$ & 2.24 & 1.57 & 1.56 & 1.41 & 0.56 & NS & NS & $*$ \\
\hline $\mathrm{C} 18: 0, \mathrm{mg} / \mathrm{g}$ & 1.38 & 1.06 & 1.08 & 1.03 & 0.29 & NS & NS & $*$ \\
\hline Cis-MUFA, mg/g & 6.47 & 7.50 & 3.96 & 5.86 & 2.40 & NS & NS & NS \\
\hline MUFA, mg/g & 6.57 & 7.59 & 4.05 & 5.69 & 2.42 & NS & NS & NS \\
\hline sum of FAs, mg/g & 12.09 & 12.15 & 8.16 & 9.52 & 3.47 & NS & NS & NS \\
\hline PUFA, mg/g & 8.24 & 9.36 & 5.38 & 6.93 & 2.78 & NS & NS & NS \\
\hline $\mathrm{SFA}, \mathrm{mg} / \mathrm{g}$ & 3.85 & 2.79 & 2.78 & 2.59 & 0.89 & NS & NS & $*$ \\
\hline $\mathrm{SFA}^{6}, \mathrm{mg} / \mathrm{g}$ & 3.10 & 2.27 & 2.05 & 2.87 & 0.50 & $*$ & $*$ & NS \\
\hline C18:0/cis-9C18:1 & 0.466 & 0.505 & 0.484 & 0.585 & 0.093 & NS & NS & $*$ \\
\hline $\mathrm{SFA} /$ cis-9C18:1 & 1.291 & 1.334 & 1.253 & 1.461 & 0.204 & NS & NS & NS \\
\hline SAF/PUFA & 0.497 & 0.301 & 0.522 & 0.382 & 0.116 & $* *$ & NS & NS \\
\hline
\end{tabular}

${ }^{1}$ weight derived from fresh organs and fresh muscle tissues

${ }^{2}$ fatty acid contents were calculated from lyophilized $M$. longissimus dorsi samples

${ }^{3}$ significance of effects: $*^{*} \mathrm{P}<0.01 ; *_{-} \mathrm{P}<0.05$; NS $-\mathrm{P} \geq 0.05$; $\mathrm{SD}$ - the pooled standard deviation

${ }^{4}$ weight of sheep after a 1 -week preliminary period (i.e. before the experiment)

${ }^{5}$ weight of sheep after 35 days of the experiment

${ }^{6}$ SFA concentration in $M$. biceps femoris of sheep

whereas the diets enriched in Se or LO with Se (LOSE) numerically decreased the level of unsaturated fatty acids. The LOSE treatment elevated the value of the ratio of cis-9C18:1 to $\mathrm{C} 18: 0$ ( $\Delta$ 9-desaturase index) and numerically increased 
the value of the ratio cis-9C18:1 to SAF. The diets enriched in LO or Se only numerically increased this value. $\mathrm{LO}$ in the diet decreased $(\mathrm{P}<0.01)$ the ratio of PUFA to SAF.

\section{DISCUSSION}

The effect of the experimental diets on organ and BW gain of the sheep were rather small, but a significant positive interaction between $\mathrm{LO}$ and Se on M. biceps femoris and BW gain of the sheep was observed. Detailed analysis of the value of ratio cis-9C18:1 to C18:0 suggest ( $\Delta 9$-desaturase index) that the experimental diets caused a reduction of $\Delta 9$-desaturation activity. The content of CLA, MUFA and PUFA numerically increased in M. longissimus dorsi of sheep fed LO probably due to stimulation of lipogenesis, elongation, and $\Delta 5-, \Delta 6$-desaturation of accumulated fatty acids. Thus feeding LO with or without Se resulted in decreasing the SFA content in M. biceps femoris. It can be hypothesized that the SE and LOSE treatment stimulated peroxidation damage and/or catabolism of fatty acids. Feeding Se with or without LO might improve the nutritional value of meats of sheep since the diets enriched with $2 \mathrm{ppm}$ of Se elevated the content of Se (an essential element) in the body of animals by $\sim 2$-fold (Arteel and Sies, 2001).

\section{CONCLUSIONS}

Adding 5\% linseed oil to the diet for 35 days improved the nutritional quality of lamb meat because it increased the content of CLA and other unsaturated FA in M. longissimus dorsi.

\section{REFERENCES}

Arteel G.E., Sies H., 2001. The biochemistry of selenium and glutathione system. Environ. Toxicol. Pharmacol. 10, 153-158

Czauderna M., Kowalczyk J., 2001. Separation of some mono-, di- and tri-unsaturated fatty acids containing 18 carbon atoms by high-performance liquid chromatography and photodiode array detection. J. Chromatogr. B 760, 165-178

Czauderna M., Kowalczyk J., 2002. HPLC separation of some unsaturated and saturated fatty acids. Chem. Anal. (Warsaw) 47, 867-882

Czauderna M., Kowalczyk J., Wąsowska I., Niedźwiedzka K.M., 2002. A highly efficient method for derivatization of fatty acids for high performance liquid chromatography. J. Anim. Feed Sci. $11,517-526$

Czauderna M., Kowalczyk J., Wąsowska I., Niedźwiedzka K.M., 2003. Determination of conjugated linoleic acid isomers by liquid chromatography and photodiode array detection. J. Anim. Feed Sci. 12, 269-382

Czauderna M., Kowalczyk J., Wąsowska I., Niedźwiedzka K.M., Pastuszewska B., 2004. Conjugated linoleic acid (CLA) content and fatty acids composition of muscle in rats fed isomers of CLA and selenium. J. Anim. Feed Sci. 13, 183-196 